\title{
Ultra-Thin Metamaterial Beam Splitters
}

\author{
Xu Zhang ${ }^{1,2}$, James E. Davis ${ }^{2}$ and Durdu Ö. Güney ${ }^{2, *(\mathbb{D}}$ \\ 1 School of Engineering and Technology, Central Michigan University, Mount Pleasant, MI 48859, USA; \\ xzhang22@mtu.edu \\ 2 Department of Electrical and Computer Engineering, Michigan Technological University, \\ Houghton, MI 49931-1295, USA; jaedavis@mtu.edu \\ * Correspondence: dguney@mtu.edu
}

Received: 27 November 2019; Accepted: 16 December 2019; Published: 19 December 2019

Featured Application: Authors are encouraged to provide a concise description of the specific application or a potential application of the work. This section is not mandatory.

\begin{abstract}
Here, we present blueprints for three types of ultra-thin beam splitters based on versatile fishnet metamaterial structures at the $1.55 \mu \mathrm{m}$ optical communication wavelength. The thicknesses of the designed polarizing beam splitter and partially polarizing beam splitter are $1 / 26$ of the free-space wavelength, while the thickness of the non-polarizing beam splitter is $1 / 13$ of the free-space wavelength. Numerical simulations show that, compared to other miniaturization approaches including popular dielectric metasurfaces, metal-based metamaterial approach can provide much thinner beam splitters with reasonable performance. Such beam splitters can enable miniaturization of conventional and advanced quantum photonic systems towards higher density, scalability, and functionality.
\end{abstract}

Keywords: metamaterial; beam splitter; polarization

\section{Introduction}

A beam splitter is an important optical component used to split a beam of incident light at a desired ratio into two separate beams. The beam splitters can be typically classified into three kinds according to the input and output polarizations: polarizing beam splitter (PBS), partially polarizing beam splitter (PPBS) and non-polarizing beam splitter (NPBS). In the recent works, NPBS based on phase gradient dielectric metasurfaces was theoretically proposed at visible and near-infrared spectra with $\mathrm{TiO}_{2}$ nanopillars on a glass substrate and $\mathrm{LiNbO}_{3}$ nanocylinders on a fused quartz substrate [1,2], and experimentally realized with a 1-bit coding scheme [3] and tested with variable splitting ratios and broadband response [4] at $\mathrm{THz}$ frequencies using Si pillars on a Si substrate fabricated by combined photolithography and deep reactive ion etching. A PBS for circularly polarized light mimicking a Rochon prism was realized with a dielectric metasurface at near-infrared wavelengths using Si nanoblocks on a fused silica substrate fabricated by series of processes including chemical vapor deposition, electron-beam evaporation, electron-beam lithography and plasma etching [5,6]. Spatially dispersive hyperbolic metamaterials were theoretically proposed to realize a PBS for white light with a wide angular range. However, the theoretical analysis therein does not consider the optical losses and discrete unit cells in the metamaterials [7]. Hyperbolic metamaterials are typically fabricated as either layered metal-dielectric structures or metallic nanowire arrays [8]. 
In earlier works, PBS designs based on an Ag mirror embedded in silica grating [9] and perforated GaAs photonic crystal [10] were theoretically proposed and experimentally realized with gratings etched in a $\mathrm{TiO}_{2}$ layer on a glass substrate fabricated by electron-beam writing and reactive ion etching [11]. Other interesting designs that were demonstrated include a PBS cube for arbitrary orthogonal polarizations based on liquid crystal polymer layers [12] and electrically tunable beam splitters employing polymer dispersed liquid crystals [13].

One can design a PBS to split light into reflected transverse electric (TE)/transverse magnetic (TM) polarization and transmitted TM/TE-polarization. Here TE-polarized means electric field is perpendicular to the plane of incidence, and TM-polarized means electric field is parallel with the plane of incidence. PBS can split unpolarized light at a 50/50 ratio, which makes it an essential element in applications, such as polarization based imaging system [14], free-space optical switching networks [15], and read-write magneto-optical data storage systems [16]. NPBS can split the light at a specific reflectance (R)/transmittance (T) ratio, at the same time, the original polarization state of the incident light is still maintained. NPBS is widely used in interferometers. Between polarizing and non-polarizing is PPBS. For example, an important application of PPBS is in quantum information processing in that it is a key component to realize the quantum controlled-NOT (CNOT) gate [17], an essential gate in the construction of a photonic quantum computer. By using a combination of the CNOT gate and single qubit rotations, one can build any quantum circuit to an arbitrary degree of accuracy [18]. NPBS can also be used in quantum entanglement distillation tasks $[19,20]$.

Inspired by the sub-wavelength unit cell size and arbitrarily controlled electromagnetic response, metamaterial-based beam splitters are promising to form compact and functional photonic devices. Here, we present three types of ultra-thin beam splitters under different geometries of fishnet metamaterial structures (see Figure 1). These beam splitters are all designed to operate at the $1.55 \mu \mathrm{m}$ wavelength, relevant to optical communication systems, where the absorption of glass material used in fiber is small. The fishnet metamaterial structures can be fabricated by standard electron-beam lithography and lift-off processes [21] or focused ion-beam milling [22]. The major advantage of the fishnet metamaterial is that none of the above mentioned beam splitters can offer designs with deep subwavelength thicknesses. The dielectric metasurfaces typically require much larger thicknesses to achieve the necessary phase gradients. Although an ultra-thin PBS based on embedded metal nanowires considering self-assembling deposition techniques [23] was proposed [24] at larger infrared wavelengths using Maxwell-Garnett theory, it has not been addressed if the performance and ultra-thin properties of the PBS are maintained close to our operating wavelength of designed fishnet structures at $1.55 \mu \mathrm{m}$. Ultra-thin beam splitters can be utilized in the miniaturization of many bulky classical and quantum photonic devices and systems. For example, the miniaturization can improve the scalability and efficiency of photonic quantum computing and communication systems [25]. 
(a)

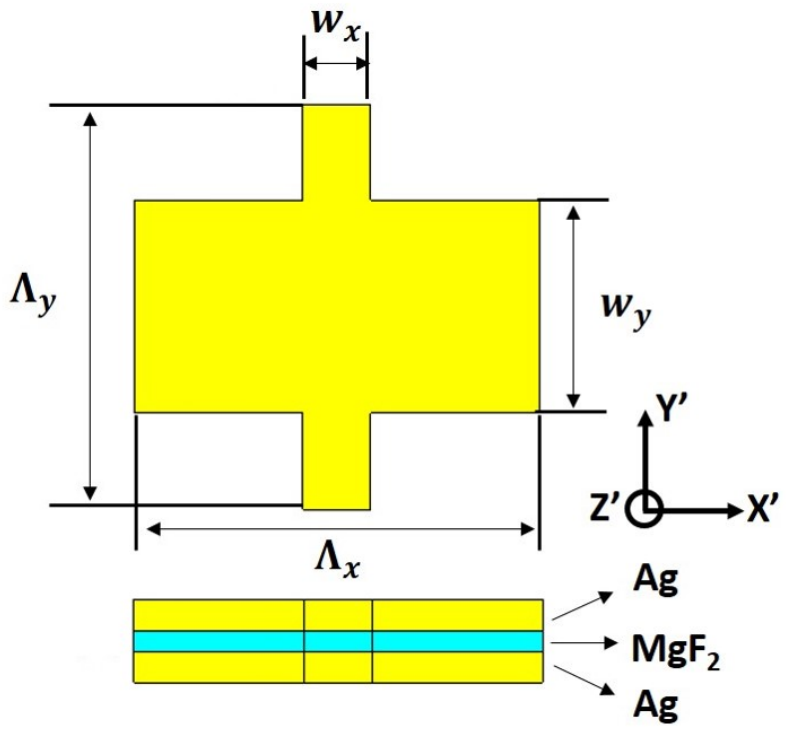

Figure 1. Schematic of the (a) unit cell of a fishnet structure with the geometric parameters indicated.

(b) Side view of the structure showing material layers.

The reflectance and transmittance for the TE-polarized and TM-polarized light can be controlled in anisotropic fishnet metamaterial structures by changing primarily the wire widths and the periods along different directions as well as metal layer thickness. Away from the resonance, with the increasing wire widths, the reflectance can be increased, while the transmittance decreases. This is because the impedance mismatch increases with the increased metal filling ratio. However, due to the anisotropy, reflectance and transmittance for different polarizations do not change at the same rate. This makes it possible to have different values of reflectance and transmittance for different polarizations. The filling ratio, hence the impedance mismatch and resultant reflectance and transmittance for different polarizations, can also be controlled similarly by the periods and metal layer thickness in the fishnet structure. On the other hand, around the resonance frequency, a rapid change occurs in the reflectance and transmittance spectra at the expense of increased absorbance. By tuning the resonance frequency, this spectral region can be shifted and thus allow for different values of reflectance and transmittance at a fixed wavelength. The resonance frequency can be shifted by adjusting the effective circuit inductance and capacitance through geometric parameters as prescribed in [26]. In the regions close to the resonance frequency, the bulk skin depth technique in $[27,28]$ can be useful in improving the efficiency.

\section{Fishnet Metamaterial Design Parameters}

We design different beam splitters based on the fishnet metamaterial in [29]. The unit cell as shown in Figure 1 consists of three layers made by $\mathrm{Ag}-\mathrm{MgF}_{2}-\mathrm{Ag}$. The thicknesses of $\mathrm{Ag}$ layer and $\mathrm{MgF}_{2}$ layer are $t_{\mathrm{Ag}}$ and $t_{\mathrm{MgF}_{2}}$, respectively. The wire widths of the fishnet structure along the $x$ and $y$ directions are $w_{x}$ and $w_{y}$, respectively. The periods are $\Lambda_{x}$ and $\Lambda_{y}$ and the structure is embedded in air. The Drude model is used to describe the permittivity of $\mathrm{Ag}$,

$$
\epsilon_{A g}=1-\frac{\omega_{p}^{2}}{\omega\left(\omega+i \omega_{c}\right)}
$$


where the plasma frequency is $\omega_{p}=1.37 \times 10^{16} \mathrm{rad} / \mathrm{s}$ and the collision frequency is $\omega_{c}=8.5 \times 10^{13} \mathrm{rad} / \mathrm{s}$ [21]. The refractive index of $\mathrm{MgF}_{2}$ is assumed to be 1.38 around $1.55 \mu \mathrm{m}$ wavelength based on a fabricated and experimentally tested fishnet metamaterial structure [21]. In the following sections, we will show different beam splitter designs by changing the geometry of the fishnet structures.

\section{Polarizing Beam Splitter}

By properly tuning the transmittance and reflectance, PBS is obtained. This is achieved when the geometric parameters are $w_{x}=400 \mathrm{~nm}, w_{y}=50 \mathrm{~nm}, \Lambda_{x}=600 \mathrm{~nm}, \Lambda_{y}=600 \mathrm{~nm}, t_{A g}=15 \mathrm{~nm}$, and $t_{\mathrm{MgF}_{2}}=30 \mathrm{~nm}$. The electromagnetic wave is incident to the fishnets at $45^{\circ}$ oblique incidence. Figure 2 shows the electric field distribution of the incident field, reflected field and transmitted field, respectively. TM-polarized light ( $E_{x}$ component) is transmitted as shown in Figure $2 \mathrm{a}$, and TE-polarized light $\left(E_{y}\right.$ component) is reflected as shown in Figure $2 \mathrm{~b}$. The designed fishnet structure achieves the goal of polarization splitting.

To explore the performance of this PBS, extinction ratio and insertion loss are calculated by

$$
\left\{\begin{array}{l}
E R_{r}=10 \log _{10}\left(\frac{R_{T E}}{R_{T M}}\right), \\
E R_{t}=10 \log _{10}\left(\frac{T_{T M}}{T_{T E}}\right),
\end{array}\right.
$$

and

$$
\left\{\begin{array}{l}
I L_{r}=10 \log _{10}\left(\frac{1}{R_{T E}}\right), \\
I L_{t}=10 \log _{10}\left(\frac{1}{T_{T M}}\right),
\end{array}\right.
$$

respectively. The extinction ratio for the TE-polarization $E R_{r}$ is the ratio of the TE-polarization reflectance $\left(R_{T E}\right)$ to the TM-polarization reflectance $\left(R_{T M}\right)$ in logarithmic scale. Similarly, the extinction ratio for the TM-polarization $E R_{t}$ is the ratio of the TM-polarization transmittance $\left(T_{T M}\right)$ to the TE-polarization transmittance $\left(T_{T E}\right)$ in logarithmic scale. Then the results are $E R_{r}=22.5 \mathrm{~dB}, E R_{t}=14.5 \mathrm{~dB}, I L_{r}=0.25 \mathrm{~dB}$, and $I L_{t}=0.26 \mathrm{~dB}$. Compared with other PBS designs [9-11], these values indicate the good performance of our designed PBS. For example, experimental results for the $\mathrm{PBS}$ with gratings etched in a $\mathrm{TiO}_{2}$ layer indicated more than $80 \%$ diffraction efficiency and above $20 \mathrm{~dB}$ extinction ratio [11]. The perforated GaAs photonic crystal based PBS design [10] was shown to have a theoretical extinction ratio of around $20 \mathrm{~dB}$. The reflecting PBS design based on a Ag mirror embedded in silica grating [9] can theoretically give around $95 \%$ diffraction efficiency and high extinction ratios up to $60 \mathrm{~dB}$ for some diffraction orders. However, an extinction ratio $>20 \mathrm{~dB}$ is already considered sufficiently a good ratio [9].

(a)

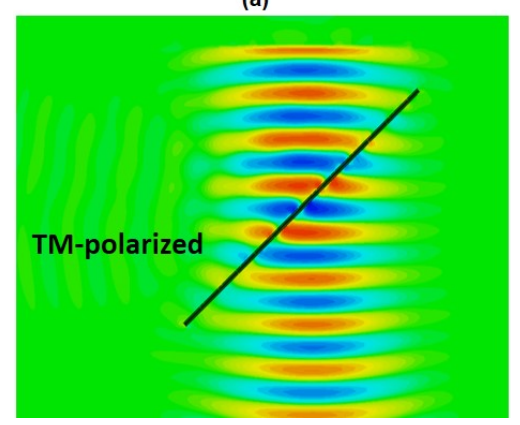

(b)

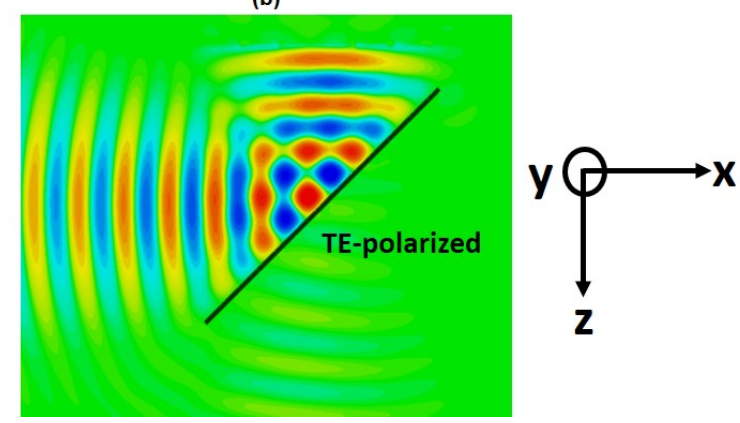

Figure 2. (a) x-component of Electric field $E_{x}$ and (b) y-component of electric field $E_{y}$ at $1.55 \mu \mathrm{m}$. The fields are incident from the $-z$ direction. 
Following the retrieval procedure described in ref [29], we can calculate the effective impedance of this beam splitter under different polarization incidence. Figure $3 a, b$ show the effective impedance $\left(Z=Z^{\prime}+i Z^{\prime \prime}\right)$ under the TE-polarized light and TM-polarized light incidence, respectively. Under the TE-polarized incidence, the real part of effective impedance is almost 0 around $1.55 \mu \mathrm{m}$, while the impedance of the background material air is 1 . So the large impedance mismatch leads to $R_{T E}=0.94$. In contrast, when the TM-polarized light is incident to the structure, the real part of the effective impedance is 0.2 around $1.55 \mu \mathrm{m}$. The impedance mismatch is reduced, so that $94 \%$ of the power is transmitted. Meanwhile, we should note that the inherently lossy metal components (i.e., $2.3 \%$ and $5.3 \%$ absorbance (A) in metallic regions for the TE-polarized and TM-polarized light, respectively) in the metamaterials result in nonzero insertion loss (see Figure 3c). Since the absorption increases around the resonance, it is desirable to be sufficiently away from the resonance for an efficient beam splitter.
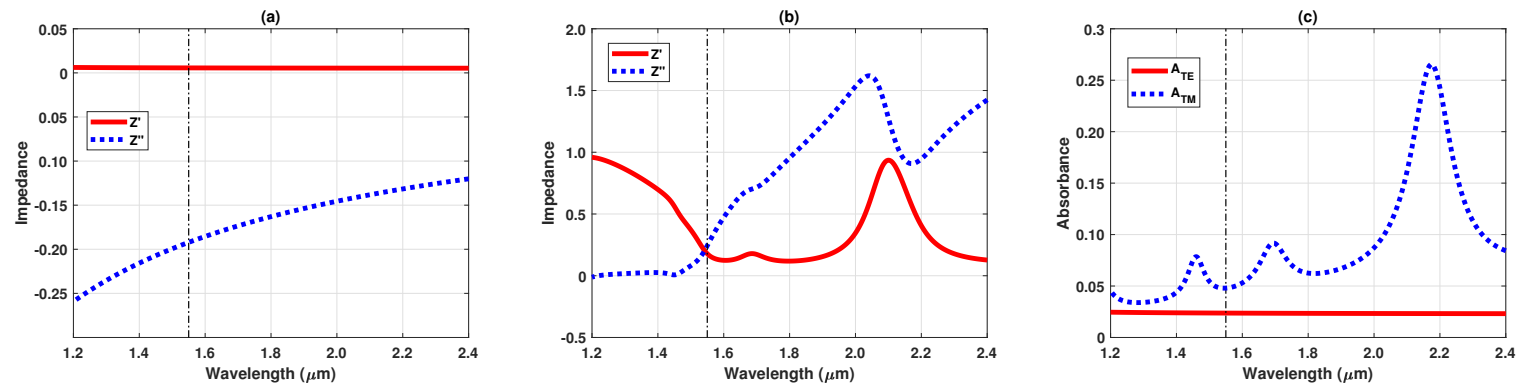

Figure 3. Effective impedance under (a) TE-polarized and (b) effective impedance under TM-polarized incident light. (c) Absorbance spectra under TE-polarized and TM-polarized light. Vertical dashed lines indicate the at $1.55 \mu \mathrm{m}$ operating wavelength of the PBS.

\section{Partially Polarizing Beam Splitter}

In this section, to further illustrate the versatility of metamaterials in designing on-demand beam splitters, we present an example PPBS useful in constructing a common linear optical quantum CNOT gate [17]. The desired splitting ratios for such an ideally lossless PPBS are the followings: $R_{T E}=1$, $R_{T M}=1 / 3$, and $T_{T M}=2 / 3$. We begin with the geometry of PBS and then optimize the structure to satisfy the requirement. The geometric parameters to obtain the required PPBS are $w_{x}=260 \mathrm{~nm}, w_{y}=140 \mathrm{~nm}$, $\Lambda_{x}=600 \mathrm{~nm}, \Lambda_{y}=560 \mathrm{~nm}, t_{A g}=15 \mathrm{~nm}$, and $t_{M_{g} F_{2}}=30 \mathrm{~nm}$. The results calculated through simulations are $R_{T E}=0.75, T_{T E}=0.02$, and $R_{T M}=0.25, T_{T M}=0.5$. Since there is always absorbance inside the structure, we cannot obtain the lossless transmittance and reflectance. However, we need to point out that in order to achieve the same probability output under different polarization, the transmittance and reflectance for TE and TM polarization should scale down with the same scale factor from the ideal case, respectively. For example, the specific PPBS design here ideally requires approximately $67 \%$ transmittance for the TM-polarized light. If this transmittance is not achieved due to absorbance, then all the reflectance and transmittance values must be scaled by the same factor. This inevitably introduces some inefficiency in the design. Once the reflectances and transmittances are scaled proportionally considering those losses, it can be seen that the largest disagreement with the ideal PPBS is only $2 \%$, which corresponds to $T_{T E}$.

\section{Non-Polarizing Beam Splitter}

Polarization-independent behavior of the NPBS can be achieved by the same length of wires (i.e., $w_{x}=w_{y}$ ), and the same period (i.e., $\Lambda_{x}=\Lambda_{y}$ ). We can obtain arbitrary splitting ratio by changing $w_{x}$ and $\Lambda_{x}$. Figure 4 shows the reflectance, transmittance, and absorbance plot for different polarizations as a function of different geometric parameters at $1.55 \mu \mathrm{m}$. The thickness of $\mathrm{Ag}$ and $\mathrm{MgF}_{2}$ are $t_{A g}=45 \mathrm{~nm}$ and 
$t_{M g F_{2}}=30 \mathrm{~nm}$, respectively. Figure 4 a shows the results by changing $w_{x}$ at $\Lambda_{x}=600 \mathrm{~nm}$. The structure exhibits almost polarization-independent behaviour when $100 \mathrm{~nm}<w_{x}<200 \mathrm{~nm}$. Figure $4 \mathrm{~b}$ shows the results by changing $\Lambda_{x}$ at $w_{x}=100 \mathrm{~nm}$. In this case, the structure is almost polarization-independent when $500 \mathrm{~nm}<\Lambda_{x}<600 \mathrm{~nm}$. We should notice that the absorbance is always small, less than 0.05 under most of the geometries, which indicates a good efficiency. The large range of reflectance and transmittance provides the opportunity to use this NPBS under different application requirements.

(a)

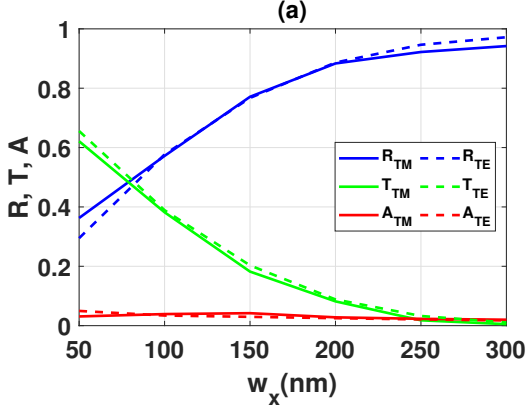

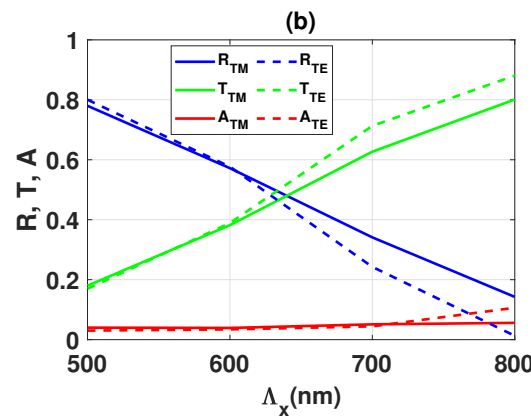

Figure 4. R, T, and A spectra versus (a) $w_{x}$, and (b) $\Lambda_{x}$ for TE-polarized and TM-polarized light.

\section{Conclusions}

In summary, we presented blueprints for three types of ultra-thin beam splitters by changing the geometric parameters of a fishnet metamaterial structure. The achieved wavelength-to-thickness ratio is $\lambda / a=26$ for the PBS and PPBS and $\lambda / a=13$ for the NPBS at $1.55 \mu \mathrm{m}$. The designed beam splitters can be integrated with other components for compact optical and photonic devices and systems. The tunability of the designed fishnet based beam splitters can be possible through, for example, voltage-tunable nematic liquid crystals [30], thermally-tunable property of physical systems with nematic liquid crystal mixtures [31], electro-optically tunable liquid crystals [13,32,33], and temperature using an external laser [34]. There exist theoretically proposed [35,36] and experimentally realized [37-39], even around $1.55 \mu \mathrm{m}$ wavelength [38], tunable fishnet metamaterials based on liquid crystals [35,37-39] and temperature [36]. Although dielectric metasurfaces were generally proposed for the miniaturization of optical components, it is difficult with dielectric metasurfaces to achieve ultra-thin beam splitters such as those presented in this work. Furthermore, even though, here, we only considered power transmittance and reflectance, plasmonic metamaterials (e.g., fishnet structures) or plasmonic metasurfaces [20,40,41] at optical wavelengths may also enable tailoring of complex reflection and transmission coefficients with ultra-thin interaction lengths. This can be especially useful for manipulating coherent optical signals including quantum information processing tasks [42].

Author Contributions: Conceptualization, D.Ö.G.; formal analysis, D.Ö.G., X.Z. and J.E.D.; investigation, X.Z. and J.E.D.; methodology, D.Ö.G., X.Z. and J.E.D.; project administration, D.Ö.G.; resources, D.Ö.G. and X.Z.; supervision, D.Ö.G.; writing—original draft, X.Z.; writing—review \& editing, D.Ö.G. and J.E.D. All authors have read and agreed to the published version of the manuscript.

Acknowledgments: Portions of this work were presented in [43].

Conflicts of Interest: The authors declare no conflict of interest. 


\section{References}

1. Ozer, A.; Yilmaz, N.; Kocer, H.; Kurt, H. Polarization-insensitive beam splitters using all-dielectric phase gradient metasurfaces at visible wavelengths. Opt. Lett. 2018, 43, 4350. [CrossRef] [PubMed]

2. Zhang, D.; Ren, M.; Wu, W.; Gao, N.; Yu, X.; Cai, W.; Zhang, X.; Xu, J. Nanoscale beam splitters based on gradient metasurfaces. Opt. Lett. 2018, 43, 267. [CrossRef] [PubMed]

3. Xing, X.; Li, Y.; Lu, Y.; Zhang, W.; Zhang, X.; Han, J.; Zhang, W. Terahertz metamaterial beam splitters based on untraditional coding scheme. Opt. Express 2019, 27, A1627-A1635. [CrossRef] [PubMed]

4. Wei, M.; Xu, Q.; Wang, Q.; Zhang, X.; Li, Y.; Gu, J.; Tian, Z.; Zhang, X.; Han, J.; Zhang, W. Broadband non-polarizing terahertz beam splitters with variable split ratio. Appl. Phys. Lett. 2017, 111, 071101. [CrossRef]

5. Wang, B.; Dong, F.; Feng, H.; Yang, D.; Song, Z.; Xu, L.; Chu, W.; Gong, Q.; Li, Y. Rochon-prism-like planar circularly polarized beam splitters based on dielectric metasurfaces. ACS Photonics 2018, 5, 1660. [CrossRef]

6. Li, Q.-T.; Dong, F.; Wang, B.; Gan, F.; Chen, J.; Song, Z.; Xu, L.; Chu, W.; Xiao, Y.-F.; Gong, Q.; et al. Polarization-independent and high-efficiency dielectric metasurfaces for visible light. Opt. Express 2016, 24, 16309-16319. [CrossRef]

7. Liu, Z.; Guo, J.; Tian, B.; Bian, Y.; Zhang, R.-Y.; Wang, Z. Omnidirectional polarization beam splitter for white light. Opt. Express 2019, 27, 7673. [CrossRef]

8. Poddubny, A.; Iorsh, I.; Kivshar, Y. Hyperbolic metamaterials. Nat. Photonics 2013, 7, 958-967. [CrossRef]

9. Zheng, J.; Zhou, C.; Feng, J.; Cao, H.; Lu, P. A metal-mirror-based reflecting polarizing beam splitter. J. Opt. A 2008, 11, 015710. [CrossRef]

10. Liu, T.; Zakharian, A.R.; Fallahi, M.; Moloney, J.V.; Mansuripur, M. Design of a compact photonic-crystal-based polarizing beam splitter. IEEE Photonics Tech. Lett. 2005, 17, 1435-1437.

11. Lalanne, P.; Hazart, J.; Chavel, P.; Cambril, E.; Launois, H. A transmission polarizing beam splitter grating. J. Opt. A 1999, 1, 215. [CrossRef]

12. Miller, S.; Tu, X.; Jiang, L.; Pau, S. Polarizing beam splitter cube for circularly and elliptically polarized light. Opt. Express 2019, 27, 16258-16270. [CrossRef] [PubMed]

13. Riquelme, M.; Ortuño, M.; MÃ irquez, A.; Gallego, S.; Pascual, I.; Beléndez, A. A dynamic beam splitter using polymer dispersed liquid crystal materials. Proc. SPIE 2012, 8498. [CrossRef]

14. Wolff, L.B. Polarization camera for computer vision with a beam splitter. J. Opt. Soc. Am. A 1994, 11, $2935-2945$. [CrossRef]

15. McCormick, F.B.; Tooley, F.A.P.; Cloonan, T.J.; Brubaker, J.L.; Lentine, A.L.; Morrison, R.L.; Hinterlong, S.J.; Herron, M.J.; Walker, S.L.; Sasian, J.M. Experimental investigation of a free-space optical switching network by using symmetric self-electro-optic-effect devices. Appl. Opt. 1992, 31, 5431-5446. [CrossRef]

16. Ojima, M.; Saito, A.; Kaku, T.; Ito, M.; Tsunoda, Y.; Takayama, S.; Sugita, Y. Compact magnetooptical disk for coded data storage. Appl. Opt. 1986, 25, 483-489. [CrossRef]

17. Chen, J.; Altepeter, J.B.; Medic, M.; Lee, K.F.; Gokden, B.; Hadfield, R.H.; Nam, S.W.; Kumar, P. Demonstration of a quantum controlled-NOT gate in the telecommunications band. Phys. Rev. Lett. 2008, 100, 133603. [CrossRef]

18. Nielsen, M.A.; Chuang, I. Quantum Computation and Quantum Information; Cambridge University Press: Cambridge, UK, 2000.

19. al Farooqui, M.A.; Breeland, J.; Aslam, M.I.; Sadatgol, M.; Ozdemir, S.K.; Tame, M.; Yang, L.; Guney, D.O. Quantum entanglement distillation with metamaterials. Opt. Express 2015, 23, 17941. [CrossRef]

20. Asano, M.; Bechu, M.; Tame, M.; Ozdemir, S.K.; Ikuta, R.; Guney, D.O.; Yamamoto, T.; Yang, L.; Wegener, M.; Imoto, N. Distillation of photon entanglement using a plasmonic metamaterial. Sci. Rep. 2015, 5, 18313. [CrossRef]

21. Dolling, G.; Wegener, M.; Linden, S. Realization of a three-functional-layer negative-index photonic metamaterial. Opt. Lett. 2007, 32, 551. [CrossRef]

22. Valentine, J.; Zhang, S.; Zentgraf, T.; Ulin-Avila, E.; Genov, D.A.; Bartal, G.; Zhang, X. Three-dimensional optical metamaterial with a negative refractive index. Nature 2008, 455, 376-379. [CrossRef] [PubMed] 
23. Li, W.-Z.; Wei, W.; Chen, J.-Y.; He, J.-X.; Xue, S.-N.; Zhang, J.; Liu, X.; Li, X.; Fu, Y.; Jiao, Y.-H.; et al. Stirring-assisted assembly of nanowires at liquidâsolid interfaces. Nanotechnology 2013, 24, 105302. [CrossRef] [PubMed]

24. Larciprete, M.C.; Centini, M.; Voti, R.L.; Bertolotti, M.; Sibilia, C. Aligned Ag nanowires for radiation manipulation: Efficient and broadband infrared polarizing beam splitter. J. Mod. Opt. 2014, 61, 1261. [CrossRef]

25. Davies, J.T.; Rickerd, C.; Grimes, M.; Guney, D.O. An n-bit general implementation of Shor's quantum period-finding algorithm. Quantum Inf. Comp. 2016, 16, 700.

26. Kafesaki, M.; Tsiapa, I.; Katsarakis, N.; Koschny, T.; Soukoulis, C.M.; Economou, E.N. Left-handed metamaterials: The fishnet structure and its variations. Phys. Rev. B 2007, 75, 235114. [CrossRef]

27. Guney, D.O.; Koschny, T.; Soukoulis, C.M. Reducing ohmic losses in metamterials by geometric tailoring. Phys. Rev. B 2009, 80, 125129. [CrossRef]

28. Vora, A.; Gwamuri, J.; Pala, N.; Kulkarni, A.; Pearce, J.M.; Guney, D.O. Exchanging Ohmic losses in metamaterial absorbers with useful optical absorption for photovoltaics. Sci. Rep. 2014, 4, 4901. [CrossRef]

29. Menzel, C.; Rockstuhl, C.; Paul, T.; Lederer, F.; Pertsch, T. Retrieving effective parameters for metamaterials at oblique incidence. Phys. Rev. B 2008, 77, 195328. [CrossRef]

30. Kowerdziej, R.; Krupka, J.; Nowinowskiâ Kruszelnicki, E.; Olifierczuk, M.; Parka, J. Microwave complex permittivity of voltage-tunable nematic liquid crystals measured in high resistivity silicon transducers. Appl. Phys. Lett. 2013, 102, 102904. [CrossRef]

31. Kowerdziej, R.; Garbat, K.; Walczakowski, M. Nematic liquid crystal mixtures dedicated to thermally tunable terahertz devices. Liq. Cryst. 2018, 45, 1040-1046. [CrossRef]

32. Zografopoulos, D.C.; Beccherelli, R.; Kriezis, E.E. Beam-splitter switches based on zenithal bistable liquid-crystal gratings. Phys. Rev. E 2014, 90, 042503. [CrossRef] [PubMed]

33. Khoo, I.C. Nonlinear optics, active plasmonic and tunable metamaterials with liquid crystals. Progr. Quantum Electron. 2014, 38, 77-117. [CrossRef]

34. Uriri, S.A.; Tashima, T.; Zhang, X.; Asano, M.; Bechu, M.; Guney, D.O.; Yamamoto, T.; Ozdemir, S.K.; Wegener, M.; Tame, M.S. Active control of a plasmonic metamaterial for quantum state engineering. Phys. Rev. A 2018, 97, 053810. [CrossRef]

35. Minovich, A.; Neshev, D.N.; Powell, D.A.; Shadrivov, I.V.; Kivshar, Y.S. Tunable fishnet metamaterials infiltrated by liquid crystals. Appl. Phys. Lett. 2010, 96, 193103. [CrossRef]

36. Hien, N.T.; Le, L.N.; Trang, P.T.; Tung, B.S.; Viet, N.D.; Duyen, P.T.; Thang, N.M.; Viet, D.T.; Lee, Y.; Lam, V.D.; et al. Characterizations of a thermo-tunable broadband fishnet metamaterial at THz frequencies. Comput. Mater. Sci. 2015, 103, 189-193. [CrossRef]

37. Zhang, F.; Zhang, W.; Zhao, Q.; Sun, J.; Qiu, K.; Zhou, J.; Lippens, D. Electrically controllable fishnet metamaterial based on nematic liquid crystal. Opt. Express 2011, 19, 1563-1568. [CrossRef]

38. Minovich, A.; Farnell, J.; Neshev, D.N.; McKerracher, I.; Karouta, F.; Tian, J.; Powell, D.A.; Shadrivov, I.V.; Tan, H.H.; Jagadish, C.; et al. Liquid crystal based nonlinear fishnet metamaterials. Appl. Phys. Lett. 2012, 100, 121113. [CrossRef]

39. Chang, C.-L.; Wang, W.-C.; Lin, H.-R.; Hsieh, F.J.; Pun, Y.-B.; Chan, C.-H. Tunable terahertz fishnet metamaterial. Appl. Phys. Lett. 2013, 102, 151903. [CrossRef]

40. Aslam, M.I.; Guney, D.O. Surface plasmon driven scalable low-loss negative-index metamaterial in the visible spectrum. Phys. Rev. B 2011, 84, 195465. [CrossRef]

41. Aslam, M.I.; Guney, D.O. Dual band double-negative polarization independent metamaterial for the visible spectrum. J. Opt. Soc. Am. B 2012, 29, 2839. [CrossRef] 
42. Tashima, T.; Ozdemir, S.K.; Yamamoto, T.; Koashi, M.; Imoto, N. Local expansion of photonic W state using a polarization-dependent beamsplitter. New J. Phys. 2009, 11, 023024. [CrossRef]

43. Zhang, X. Controlling Light with Metadevices. Ph.D. Thesis, Michigan Technological University, Houghton, MI, USA, 2018. 